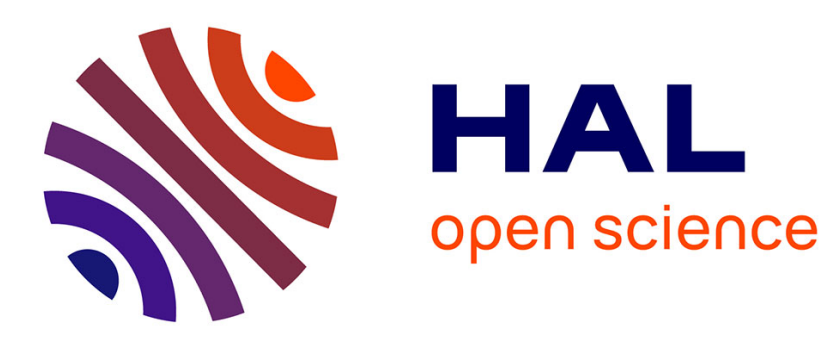

\title{
Contribution à la théorie de l'ionisation par un champ électrique, en régime Poole
}

\author{
Alain Pillonnet, Roger Ongaro
}

\section{To cite this version:}

Alain Pillonnet, Roger Ongaro. Contribution à la théorie de l'ionisation par un champ électrique, en régime Poole. Revue de Physique Appliquée, 1989, 24 (1), pp.109-116. 10.1051/rphysap:01989002401010900 . jpa-00246019

\section{HAL Id: jpa-00246019 https://hal.science/jpa-00246019}

Submitted on 1 Jan 1989

HAL is a multi-disciplinary open access archive for the deposit and dissemination of scientific research documents, whether they are published or not. The documents may come from teaching and research institutions in France or abroad, or from public or private research centers.
L'archive ouverte pluridisciplinaire HAL, est destinée au dépôt et à la diffusion de documents scientifiques de niveau recherche, publiés ou non, émanant des établissements d'enseignement et de recherche français ou étrangers, des laboratoires publics ou privés. 


\title{
Contribution à la théorie de l'ionisation par un champ électrique, en régime Poole
}

\author{
Alain Pillonnet et Roger Ongaro \\ Laboratoire d'Electricité, Université Claude Bernard Lyon 1, 43 boulevard du 11 novembre 1918, \\ 69622 Villeurbanne Cedex, France
}

(Reçu le 5 avril 1988, révisé le 27 juin 1988, accepté le 12 septembre 1988)

\begin{abstract}
Résumé. - On rappelle les approches théoriques existantes de l'action d'un champ électrique en régime Poole, sur des paires de puits coulombiens. Une analyse comparative montre l'insuffisance de certaines des hypothèses du modèle habituel. L'expression analytique de la hauteur de la barrière de potentiel extérieure à la paire, est obtenue. Il s'en déduit un effet Poole Frenkel, modifié par l'influence réciproque des puits. En conclusion, la nécessité d'une approche beaucoup plus générale est mise en évidence.

Abstract. - A brief outline is given of the existing approaches of the field induced ionization, in the so-called Poole regime, involving paired coulombic wells. A comparative study allows to put forward the inadequacy of some hypotheses of the usual model. An analytical expression is derived for the barrier height, external to the pair. The resulting Poole Frenkel effect, modified by the reciprocal influence of the wells, is computed. As a conclusion, the need for a more general approach is evidenced.
\end{abstract}

\section{Introduction.}

Historiquement la loi de Poole [1-3] a été énoncée empiriquement, afin de rendre compte de la forme des courants permanents, obtenus dans le mica lorsqu'une tension continue est appliquée. Elle indique que le courant est proportionnel à $\exp (K F), F$ étant le champ électrique appliqué, et $K$ une constante appropriée.

Cette loi est susceptible de recevoir une interprétation théorique, fondée sur les mêmes hypothèses que l'effet Poole-Frenkel (effet PF). Dans ce dernier cas, le champ appliqué abaisse le potentiel coulombien des donneurs, supposés isolés (conductivité du type n), c'est-à-dire assez éloignés pour que leurs influences mutuelles puissent être considérées comme négligeables. La densité d'électrons libérés augmente en conséquence, et la variation correspondante du courant est alors, usuellement, déterminée comme proportionnelle à $\exp (\beta \sqrt{F} / k T)$, avec $\beta=\left(e^{3} / \pi \varepsilon_{0} \varepsilon\right)^{1 / 2}$. Les auteurs traitant du régime Poole supposent que les interactions entre puits coulombiens cessent d'être négligeables, lorsque la densité des sites crôit. L'influence du champ s'en trouve alors modifiée. Il est généralement admis, que la transition entre les deux lois se produit lorsque la distance $a$, entre les sites, est égale à
$2 x_{\mathrm{m}}, x_{\mathrm{m}}=\left(e / 4 \pi \varepsilon_{0} \varepsilon F\right)^{1 / 2}$ étant l'abscisse du maximum $M$, pour des puits isolés (courbe en pointillés de la Fig. 1). Cela impliquerait, selon Vollmann [4], l'existence d'un champ de transition $F_{\mathrm{t}}=$ $N_{\mathrm{d}}^{2 / 3}(\beta / e)^{2}$, limite supérieure du régime Poole ; ou inversement, d'une densité minimale $N_{\mathrm{d}}=$ $(e \sqrt{F} / \beta)^{2}$. Celle-ci, par exemple, serait de quelques $10^{17} \mathrm{~cm}^{-3}$, sous un champ $F=10^{7} \mathrm{~V} \cdot \mathrm{m}^{-1}$, avec une constante diélectrique $\varepsilon=2,2$, égale à celle du nitrate de cellulose aux fréquences optiques [5].

Dans cet article notre propos est triple. Il consiste d'abord à analyser le modèle fondateur du régime Poole reposant, selon l'hypothèse simplificatrice de Hill [6], sur un regroupement des sites par paires, l'influence entre puits coulombiens étant purement interne à chaque paire. Il consiste en second lieu à déterminer une expression exacte de l'abaissement du potentiel externe, en amont du champ (point $\mathbf{M}^{\prime \prime}$ de la Fig. 1). Cela permettra de situer numériquement la validité de l'équivalence adoptée par Hill entre $M^{\prime \prime}$ et le point $\mathbf{M}^{\prime \prime \prime}$ maximum pour un puits coulombien unique, doublement ionisé (cf. paragraphe 3.3). Enfin, nous serons amenés à reposer sur une base élargie, le problème général de l'interaction entre des sites coulombiens, sans lui apporter toutefois, dans le cadre restreint actuel, une réponse élaborée. 


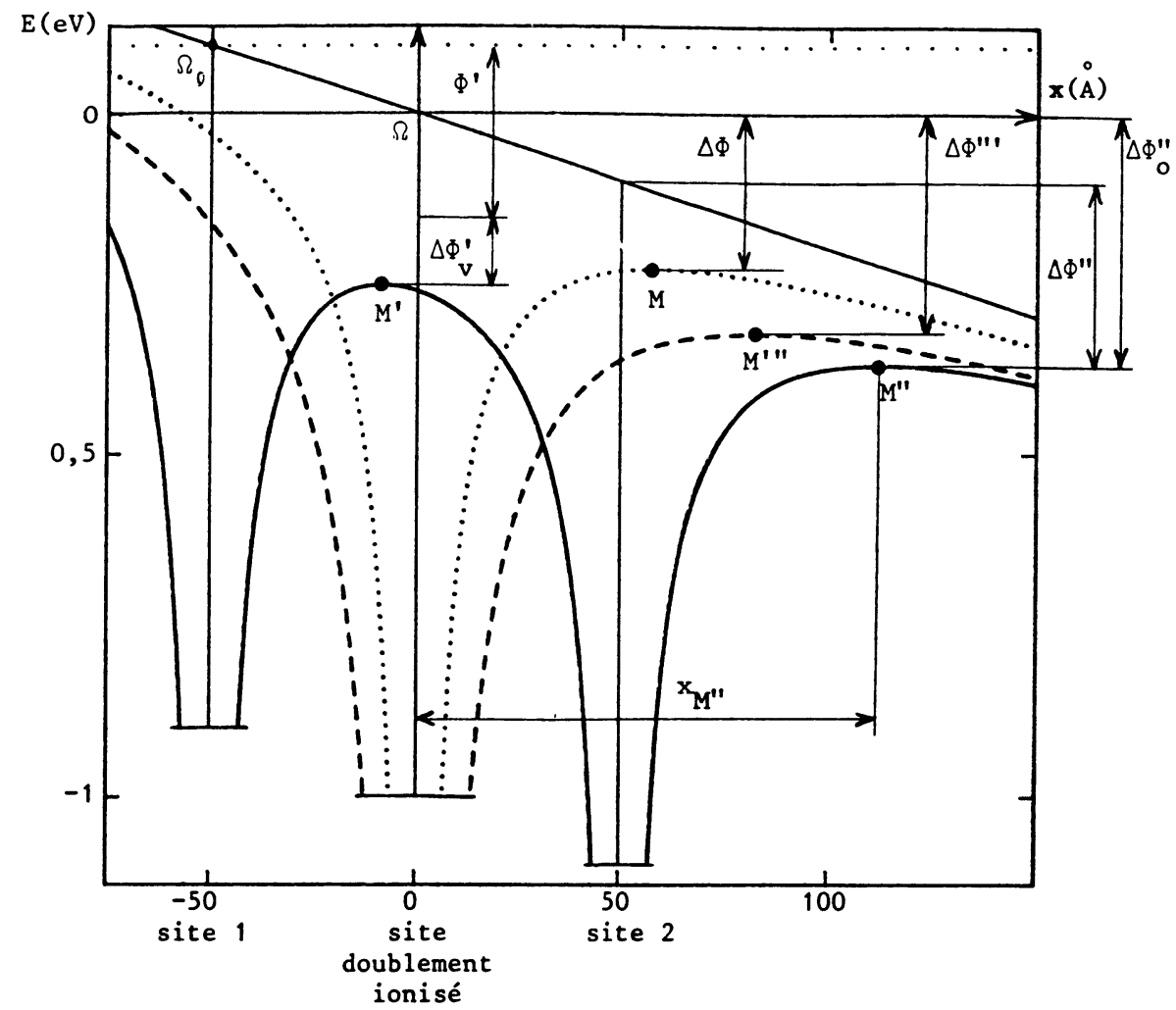

Fig. 1. - Energies potentielles : du puits coulombien doublement ionisé (- -$)$; d'une paire de puits isolée $\left(()\right.$; du puits coulombien isolé (...). $\Phi=1 \mathrm{eV} ; \varepsilon=2,2 ; a=100 \AA ; F=2 \times 10^{7} \mathrm{~V} \cdot \mathrm{m}^{-1}$.

[Potential energies : for a single coulombic well doubly ionized.(- - ) ; for an isolated pair of coulombic wells (—) ; for a single coulombic well (...). $\Phi=1 \mathrm{eV} ; \varepsilon=2.2 ; a=100 \AA ; F=2 \times 10^{7}$ V.m ${ }^{-1}$.]

\section{Description et analyse du modèle usuel.}

Le potentiel d'une paire de puits coulombiens est représenté, sous champ, par la courbe en trait plein de la figure 1. Parmi les auteurs qui ont tenté de donner une assise théorique au régime Poole, il convient de citer principalement outre Hill, Dussel et Boër [7] et Vollmann [4]. Ces auteurs admettent les hypothèses simplificatrices suivantes.

(1) Les sites, purement coulombiens, sont regroupés par paires de proches voisins, suffisamment éloignées les unes des autres pour que leurs influences mutuelles soient négligeables. Hill [6] estime cette hypothèse nécessaire car, selon lui, son abandon, d'une part obligerait à définir un potentiel coulombien écranté ; d'autre part impliquerait une évolution spatiale continue des propriétés du matériau. Ces deux justifications exigeraient un examen approfondi, qui sortirait nettement du cadre du travail actuel. Notons toutefois qu'il existe des traitements, tels ceux de Feltz et al. [8] ou de Fritzsch et Bobe [9], dans lesquels un potentiel coulombien écranté est introduit, respectivement, à partir d'une approche théorique un peu simple, ou d'une manière purement empirique.

Nous montrerons dans un prochain exposé que la considération de puits coulombiens conduit à de grandes difficultés théoriques, l'abaissement de la barrière inter-sites dépendant alors du nombre total de donneurs présents dans l'échantillon. Il faut remarquer en outre que les auteurs semblent écarter l'hypothèse du regroupement par paires pour le calcul de la densité des sites. Ils l'évaluent ainsi comme s'il s'agissait d'une répartition moyenne régulière $\left(N_{\mathrm{d}}=a^{-3}\right)$.

(2) Le premier site (site 1), en aval par rapport au sens du champ, est supposé plein, tandis que le second (site 2) est ionisé. Il faut relever l'étrangeté de cette hypothèse. La figure 1 montre en effet que la hauteur de la barrière de potentiel interne, à franchir par un électron pour qu'il s'échappe thermiquement du site 1 , est inférieure à celle que doit franchir un électron lié au site 2 pour être libéré. En conséquence, la probabilité d'occupation est normalement plus faible pour le premier site que pour le second. Au demeurant un taux d'occupation plus élevé du second site préviendrait contre une possibilité de repiégeage immédiat de l'électron libéré par le premier.

Mais cette hypothèse a l'avantage de conduire au régime Poole, en considérant seulement l'influence du second puits sur l'abaissement de la barrière interne. Dans le cas contraire où le site 1 serait vide 
et le site 2 occupé, l'apparente inversion des probabilités d'occupation serait levée. Mais alors l'ionisation du site 2 serait peu influencée par le site 1 , et conduirait à un effet de type PF (cf. paragraphe 4 cidessous). D'une manière plus générale, le problème de l'interaction entre sites pleins (neutres) ou vides (ionisés) ne semble pas avoir été analysé en profondeur. En particulier, les auteurs ne précisent pas quelle barrière de potentiel les électrons doivent franchir pour être libérés, lorsque les deux sites sont pleins. Dire qu'alors ces sites ne s'influencent pas, est une affirmation qui demande à être examinée d'une manière approfondie.

Ainsi les hypothèses (1) et (2) paraissent bien artificielles. La première permet toutefois une approche mathématique plus simple du problème de l'interaction entre les puits.

Plus généralement, on doit noter que ce modèle, adopté dans la littérature pour établir la loi de Poole, est extrêmement simplifié. En particulier, le champ est supposé uniforme, ce qui implique l'absence de charges d'espace et d'effets d'électrodes. Le problème est traité à une dimension. Il ne nous semble pas utile de le ré-examiner à trois dimensions, sans l'avoir auparavant re-situé dans un contexte plus général. Nous écartons de même l'éventualité, introduite par Hill [6], d'un effet tunnel à travers la barrière interne à la paire, ou à travers la barrière externe du site 2 . Des élargissements de ce type seront réalisés méthodiquement dans des travaux ultérieurs.

\section{Expressions théoriques du modèle de la paire.}

Avant d'amorcer un développement un peu plus complet du problème, rappelons les résultats théoriques obtenus par les auteurs.

3.1 RÉGIME POOLE « USUEL » (1 DIMENSION). - Le régime Poole le plus simple, obtenu en considérant des paires isolées de puits coulombiens alignées dans la direction du champ, est désigné, dans la suite, régime Poole « usuel». On suppose constante la distance a entre les deux composantes des paires. Dans ce modèle, une distribution désordonnée des donneurs se traduirait par exemple par des fluctuations de la distance $b$ entre les paires. L'origine des abscisses étant prise au centre du site 1 (point $\Omega_{0}$ ) de la Fig. 1), l'équation du potentiel s'écrit alors, en présence du champ appliqué :

$$
\begin{aligned}
E(x, F)= & -\frac{e^{2}}{4 \pi \varepsilon \varepsilon_{0}|x|}-\frac{e^{2}}{4 \pi \varepsilon \varepsilon_{0}|a-x|} \\
& -e F x .
\end{aligned}
$$

Une approximation couramment admise, en particulier par Hill, consiste à confondre avec $a / 2$ la position $x_{\mathrm{m}^{\prime}}$ du maximum $\mathrm{M}^{\prime}$ de $E(x, F)$ entre les puits. L'abaissement de la barrière entre les sites est alors la somme de deux termes:

$$
\frac{\beta^{2}}{e a}+\frac{1}{2} e a F=\Phi^{\prime}+\Delta \Phi^{\prime} .
$$

Le premier $\Phi^{\prime}=\beta^{2} /$ ea représente l'abaissement de la barrière interne dû à l'effet de proximité des puits, tandis que le second est l'abaissement de cette barrière par le champ.

En l'absence de champ, la probabilité d'ionisation fait ainsi intervenir la profondeur apparente du premier puits :

$$
\Phi_{a}^{\prime}=\Phi-\Phi^{\prime}
$$

Contrairement à l'usage, nous désignons par :

$$
\Delta \Phi^{\prime}=\frac{1}{2} e a F
$$

la seule contribution due au champ appliqué. Le courant permanent a alors la forme :

$$
j_{0} \propto F \exp \left(-\frac{\Phi_{a}^{\prime}}{k T}\right) \exp \alpha^{\prime}
$$

$\operatorname{avec} \alpha^{\prime}=\frac{1}{2} \frac{e a F}{k T}=\frac{\Delta \Phi^{\prime}}{k T}$.

3.2 AJUSTEMENTS DU RÉGIME POOLE «USUEL ». Selon Vollmann deux types d'ajustements peuvent être apportés à l'équation (5), selon la valeur du champ appliqué. L'auteur admet qu'aux champs $F<F_{\mathrm{t}}$, le courant est contrôlé uniquement par la barrière intersites (régime Poole). Tandis que pour $F>F_{\mathrm{t}}$ la hauteur de la barrière interne se rapproche de celle d'un site unique : le courant tend alors à être de type PF. La démonstration en est la suivante.

La position $x_{\mathrm{m}^{\prime}}$, du maximum situé entre les deux sites $\left(0<x<\frac{a}{2}\right)$, est solution d'une équation du quatrième degré que Vollmann résout par un procédé d'itération qui, en conformité avec le sens physique du modèle, exige des développements limités différents pour les champs inférieurs ou supérieurs à $F_{\mathrm{t}}$.

Pour les bas champs qui concernent le régime Poole :

$$
\begin{aligned}
& x_{\mathrm{m}^{\prime}} \cong \frac{a}{2}\left(1-\frac{1}{4}\left(\frac{a}{2 x_{\mathrm{m}}}\right)^{2}\right)=\frac{a}{2}\left(1-\frac{1}{4} \frac{F}{F_{\mathrm{t}}}\right) ; \\
& x_{\mathrm{m}^{\prime}} \leqq \frac{a}{2} .
\end{aligned}
$$

Il en déduit l'abaissement $\Delta \Phi_{\mathrm{v}}^{\prime}$ de la barrière de potentiel par le champ, avec $f=F / F_{\mathrm{t}}$ :

$$
\Delta \Phi_{v}^{\prime}=\Delta \Phi^{\prime}\left(1-\frac{1}{4} f\right)
$$


Vollmann se plaçant alors dans les conditions du modèle de Simmons [10], obtient, pour le courant, l'expression :

$$
\begin{aligned}
j_{0}=e \mu\left(N_{\mathrm{c}} N_{\mathrm{d}}\right)^{1 / 2} F & \exp \left(-\frac{\Phi_{a}^{\prime}}{2 k T}\right) \times \\
& \times \exp \left(\frac{\alpha^{\prime}}{2}\left(1-\frac{1}{4} f\right)\right)
\end{aligned}
$$

$N_{\mathrm{c}}$ et $N_{\mathrm{d}}$ étant respectivement la densité d'états dans la bande de conduction et la densité des donneurs, et $\mu$ la mobilité. Ainsi la proportionnalité à exp $\alpha^{\prime}$ est corrigée par un facteur inférieur à $1 / 4$.

Pour les champs supérieurs à $F_{\mathrm{t}}$, Vollmann donne pour valeur approchée de $x_{\mathrm{m}^{\prime}}$ :

$$
x_{\mathrm{m}^{\prime}}=x_{\mathrm{m}}\left(1-\frac{1}{8 f}\right) ; x_{\mathrm{m}} \leqq \frac{a}{2} .
$$

L'abaissement de la barrière de potentiel est alors :

$$
\Delta \Phi_{\mathrm{v}}=\beta \sqrt{F}\left(1+\frac{1}{8 f}\right)=\Delta \Phi\left(1+\frac{1}{8 f}\right) .
$$

Il obtient ainsi, en ne considérant que l'ionisation du site 1 , un courant de type $P F$, corrigé par un facteur inférieur à $1 / 8$.

3.3 Équivalence Selon Hill entre $\mathbf{M}^{\prime \prime}$ et $\mathbf{M}^{\prime \prime \prime}$. Pour les champs faibles, Hill considère aussi que seul intervient le processus d'ionisation par dessus la barrière interne. Cependant, la validité de l'approximation $x_{\mathrm{m}^{\prime}} \sim a / 2$ peut être, selon son estimation, étendue jusqu'à des champs $F<4 F_{\mathrm{t}}(f<4)$. Dans cet intervalle, l'équation (5) est alors directement utilisable.

Pour les champs élevés, Hill admet qu'un effet tunnel, à travers la barrière interne, rend plus probable la présence de l'électron sur le site 2 . Il cherche alors à déterminer l'abscisse $x_{\mathrm{m}}$ ' du maximum $\mathbf{M}^{\prime \prime \prime}$ qu'aurait à franchir l'électron pour être libéré. Il considère pour cela, que cette barrière correspond approximativement à celle d'un puits unique doublement ionisé, situé à l'abscisse $a / 2$. Alors (cf. Fig. 1) :

$$
x_{\mathrm{m}^{\prime \prime}}=\frac{\beta}{\mathrm{e} \sqrt{2 F}} \quad \text { et } \Delta \Phi^{\prime \prime \prime}=\sqrt{2} \Delta \Phi
$$

$\Delta \Phi$ étant l'abaissement de barrière par le champ, pour un puits isolé simplement ionisé.

Hill obtient ainsi, en champs élevés, un effet PF modifié par la présence du site 1 .

\section{Etude complète du modèle de la paire isolée de puits.}

4.1 DÉTERMINATION DE L'ABAISSEMENT DU POTENTIEL DU SECOND SITE. - L'approche de Hill cidessus repose sur un concept d'équivalence entre les potentiels créés par un puits coulombien deux fois ionisé, et celui de la paire doublement chargée. Une telle équivalence ne nous semblant pas fermement établie, nous avons déterminé l'expression exacte de la hauteur de barrière du site, sous un champ $F$ quelconque. La manière la plus simple, pour y parvenir, est de ré-écrire l'équation (1a) en prenant l'origine des abscisses au centre de la paire de puits (point $\Omega$ de la Fig. 1). En effet, la recherche du maximum du potentiel passe alors par la résolution d'une équation bi-carrée. Ainsi, en dérivant l'équation

$E(x, F)=-\frac{\beta^{2}}{4 e}\left(\frac{1}{\left|x-\frac{a}{2}\right|}+\frac{1}{\left|x+\frac{a}{2}\right|}\right)-e F x$

on obtient l'abscisse $x_{\mathrm{m}^{\prime \prime}}$ du maximum, qui s'exprime en valeur relative par:

$$
\xi_{\mathrm{m}^{\prime \prime}}=\frac{x_{\mathrm{m}^{\prime \prime}}}{a / 2}=\sqrt{\frac{2+\xi_{\mathrm{m}}^{2 \prime \prime}+\xi_{\mathrm{m}^{\prime \prime}} \sqrt{\xi_{\mathrm{m}}^{2}+8}}{2}}
$$

$\xi_{\mathrm{m}^{\prime \prime}}$ étant le maximum réduit $x_{\mathrm{m}^{\prime \prime}} / a / 2$, donné par (11). Cette équation s'écrit aussi en posant :

$$
\begin{aligned}
& \xi_{\mathrm{m}^{\prime \prime \prime}}^{2}=\frac{2}{F}\left(\frac{\beta}{e a}\right)^{2}=\frac{2}{f} \\
& \xi_{\mathrm{m}^{\prime \prime}}=\sqrt{\frac{1+f+\sqrt{1+4 f}}{f}} .
\end{aligned}
$$

L'abaissement de potentiel correspondant est donné par :

$$
\begin{array}{r}
\Delta \Phi_{0}^{\prime \prime}=\frac{1}{2} \beta \sqrt{F} \sqrt{1+f+\sqrt{1+4 f}} \times \\
\times \frac{3+\sqrt{1+4 f}}{1+\sqrt{1+4 f}} .
\end{array}
$$

L'abaissement du potentiel relatif au premier puits s'obtient alors en décalant les abscisses de $a / 2$ :

$$
\begin{aligned}
\Delta \Phi^{\prime \prime}=\frac{\Delta \Phi}{2} \sqrt{1+f+\sqrt{1+4 f}} \times \\
\times \frac{3+\sqrt{1+4 f}}{1+\sqrt{1+4 f}}-\frac{1}{2} e a F .
\end{aligned}
$$

Cette équation peut aussi s'écrire :

$$
\begin{aligned}
\Delta \Phi^{\prime \prime}= & \frac{1}{2} \beta \sqrt{F}(\sqrt{1+f+\sqrt{1+4 f}} \times \\
& \left.\times \frac{3+\sqrt{1+4 f}}{1+\sqrt{1+4 f}}-\sqrt{f}\right) \\
= & \beta \sqrt{F} g(f)
\end{aligned}
$$

ou

$$
\Delta \Phi^{\prime \prime}=\frac{\beta^{2}}{e a} \sqrt{f} g(f)=\Phi^{\prime} \sqrt{f} g(f) .
$$


L'équation (14b) montre clairement que l'abaissement du second puits par le champ est de type PF, modifié par une fonction $g(f)$ qui traduit l'influence du premier puits.

4.2 COMPARAISONS QUANTITATIVES DE $\Delta \Phi^{\prime \prime}$ A $\Delta \Phi^{\prime \prime \prime}$ ET $\Delta \Phi$. - Nous avons cherché à apprécier les erreurs sur $\Delta \Phi^{\prime \prime}$ qu'impliquerait : d'une part le choix de l'équivalence de Hill $\Delta \Phi^{\prime \prime \prime}$; d'autre part, le choix de l'abaissement PF usuel $\Delta \Phi$, qui suppose une influence négligeable du premier site sur le second. Pour cela, nous avons, par calcul numérique, obtenu la figure 2 , qui donne les variations en fonction de $f$ des rapports $\Delta \Phi^{\prime \prime} \angle \Delta \Phi$ (courbe (1)), et $\Delta \Phi^{\prime \prime} / \Delta \Phi^{\prime \prime \prime}=\Delta \Phi^{\prime \prime} / \sqrt{2} \Delta \Phi$ (courbe (2)). On voit ainsi que, considérer le second puits comme isolé, se traduit par une erreur limitée à $5 \%$ lorsque $f$ est supérieur à 20 environ. Inversement, l'équivalence de Hill, donne les valeurs de $\Delta \Phi^{\prime \prime}$, à $5 \%$ près, lorsque $f$ est inférieur à $2 \times 10^{-2}$ environ. Donc, entre $2 \times 10^{-2}$ et 20 , aucune des deux approximations ne semble convenable. On doit noter cependant que, jusqu'au voisinage de $f=1$, le régime Poole dû au premier puits devrait apporter la contribution principale. Ainsi le modèle hydrogénoïde doublement ionisé de Hill ne devrait jamais apparaître comme une approximation acceptable, puisqu'il est valable seulement en bas champs. Sachant que $f=\left(\mathrm{e}^{2} / \beta^{2}\right) a^{2} F$ est proportionnel à $a^{2} F$ (et à $\varepsilon)$, l'inadaptation de ce modèle apparaît d'autant plus grande que la densité des sites est plus élevée.

Nous avons cherché à savoir quel est l'intervalle de variation de $f$ à considérer, afin de discuter des limites quantitatives de $F$. Pour cela, nous prenons $\varepsilon=2,2$, valeur proche du minimum envisageable. Alors $f=3,8 \times 10^{8} a^{2} F$ est une fonction minorée de $a$ et de $F$. La distinction entre les régimes Poole et $\mathrm{PF}$, ainsi que les conditions requises pour leurs validités respectives, peuvent alors être abordées de la manière suivante.

(1) Pour que les deux puits soient définis et distincts, il faut que l'abaissement $\Phi^{\prime}$, dû à leur interaction, soit inférieur ou égal à la profondeur $\Phi$ du niveau fondamental des sites. En effet, il est difficile de donner un sens physique à la paire de puits, lorsque le maximum qui les sépare est audessous du niveau fondamental. Cela impose une limite inférieure à $a$, car :

$$
a \geqq a_{\mathrm{m}}=\frac{\beta^{2}}{e \Phi} .
$$

Ainsi, par exemple, pour $\Phi \cong 1 \mathrm{eV}, a_{\mathrm{m}}=25 \AA$. Dans les conditions numériques adoptées ici, le modèle des paires isolées où la distance moyenne $b$ entre les paires est nécessairement très supérieure à $a$, ne peut donc être éventuellement adopté, que pour des densités de donneurs très inférieures à la valeur $6,4 \times 10^{19} \mathrm{~cm}^{-3}$ obtenue avec $b=a$.

(2) Le modèle conduit à considérer, en première approximation, que l'effet PF n'intervient qu'à partir du champ $F_{\mathrm{t}}=(\beta / e a)^{2}$. Or le calcul donne, pour $a=25 \AA, F_{\mathrm{t}}=4,2.10^{8} \mathrm{~V} . \mathrm{m}^{-1}$. Dans ce cas donc, il n'y aurait plus d'effet PF puisque $F_{\mathrm{t}}$ est de l'ordre des champs maximaux admissibles $F_{\mathrm{d}}$ (champs disruptifs) dans les isolants. A l'inverse, si on

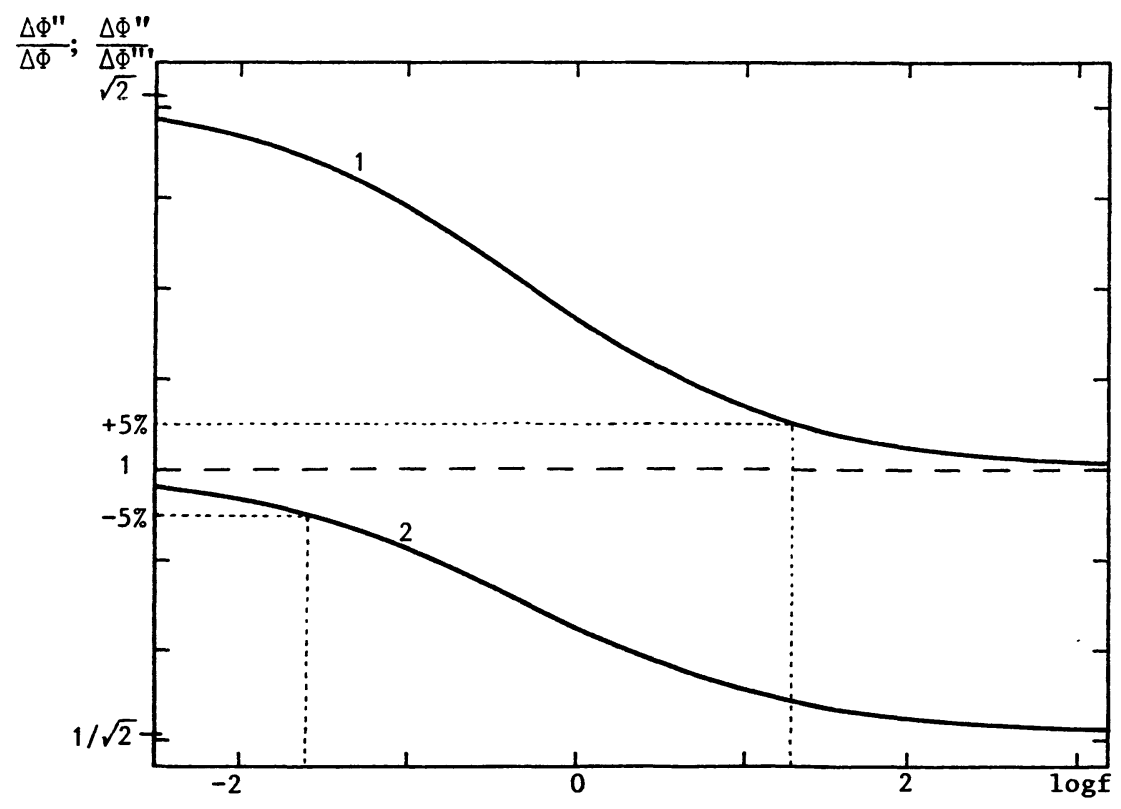

Fig. 2. - Variations en fonction de $f$, des rapports $\Delta \Phi^{\prime \prime} / \Delta \Phi$ (courbe (1)), et $\Delta \Phi^{\prime \prime} / \Delta \Phi^{\prime \prime \prime}$ (courbe (2)).

[Variations against $f$, of the ratios $\Delta \Phi^{\prime \prime} / \Delta \Phi$ (curve (1)), and $\Delta \Phi^{\prime \prime} / \Delta \Phi^{\prime \prime \prime}$ (curve (2)).] 
suppose que a ne peut guère excéder $10^{3} \AA$, on obtient $F_{\mathrm{t}} \sim 2,6 \times 10^{5} \mathrm{~V} \cdot \mathrm{m}^{-1}$. Donc, l'effet PF, associé à $\mathrm{M}^{\prime \prime \prime}$, ne peut intervenir, au maximum, que sur l'intervalle, éventuellement nul $\left(F_{\mathrm{t}}, F_{\mathrm{d}}\right)$.

(3) On peut alors admettre, pour valeur maximale de $f, f_{\max }=F_{\mathrm{d}} / F_{\mathrm{t}}$. Dans le cas où $a=10^{3} \AA$, et $\varepsilon=2,2$, on aurait donc environ $f_{\max } \sim 1600$, cette limite étant proportionnelle à $a^{2}$.

4.3 VARIATIONS COMPARÉES DES CONDUCTIVITÉS. Nous avons représenté sur la figure 3 les variations, en unités arbitraires, de la densité d'électrons libres, en fonction de $\sqrt{F}$. Les courbes (1) corres- pondent à l'effet $\mathrm{PF}$ usuel, obéissant à l'expression classique $\quad n \propto \exp \left(-\frac{\Delta \Phi}{k T}\right)=\exp \left(-\frac{\beta \sqrt{F}}{k T}\right)$. Les courbes (2) à (4) traduisent l'effet PF modifié de l'équation (14b). Elles sont obtenues pour différentes valeurs de $a$, en utilisant toujours l'expression classique dans laquelle $\Delta \Phi$ est remplacé par $\Delta \Phi^{\prime \prime}$, c'est-à-dire en écrivant $n \propto \exp \left(-\frac{\Delta \Phi^{\prime \prime}}{k T}\right)=$ $\exp \left(-\frac{\beta}{k T} \sqrt{F} g(f)\right)$. On constate ainsi que ces courbes sont assimilables à des droites sur des intervalles plus réduits, et que leur pente augmente lorsque a diminue. Finalement, le fait de tenir

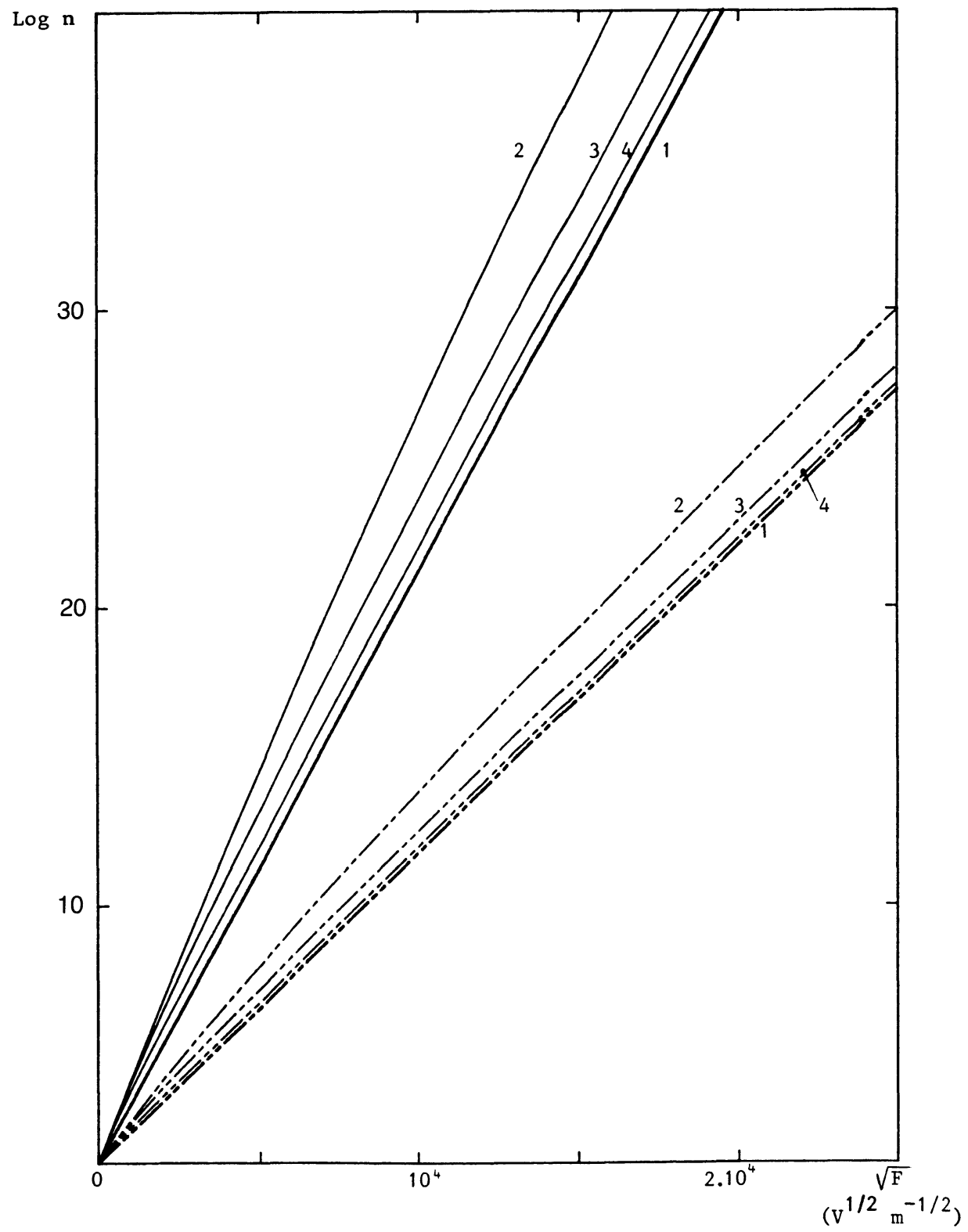

Fig. 3. - Variations, en unités arbitraires, de la densité $n$ d'électrons de conduction, en fonction de $\sqrt{F}$. (1) : effet PF

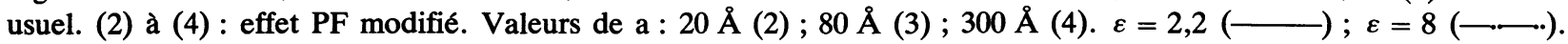

[Variations, in arbitrary units, of the density $n$ of conduction electrons, in terms of $\sqrt{F}$. (1): usual PF effect ; (2)-(4) : modified PF effect. $a$ values : $20 \AA(2) ; 80 \AA(3) ; 300 \AA(4) . \varepsilon=2.2(\longrightarrow) ; \varepsilon=8(\longrightarrow-$. $]$ 
compte de l'effet $\mathrm{PF}$ en $\mathrm{M}^{\prime \prime}$ ne conduit pas à des résultats qui donnent des valeurs de $\beta$ plus proches des valeurs ${ }^{*} \beta$ trouvées expérimentalement. En effet, les valeurs obtenues sont supérieures à celles que donne l'effet PF usuel; alors que ${ }^{*} \beta$ est généralement inférieur à cette valeur.

Les courbes obtenues sur la figure 3 ne sont cependant qu'indicatives d'une tendance générale aux champs élevés. De plus, l'effet PF n'a pas exactement la même origine selon les auteurs. Ainsi, pour Vollmann, il résulterait d'une excitation pardessus la barrière interne de la paire; tandis que pour Hill, il s'agirait d'une ionisation du site 2 pardessus la barrière extérieure, modifiée par la présence du site 1 , le site 2 étant "approvisionné » en électrons par effet tunnel interne. Cette divergence d'approche est à mettre en parallèle avec la différence d'estimation, du champ de transition, qui découle des choix de ces auteurs. Il résulte, en effet, de l'approximation $x_{\mathrm{m}^{\prime}} \cong a / 2$, faite par Hill, que la valeur qu'on pourrait en déduire pour ce champ serait le quadruple de celle donnée par Vollmann.

Mais, plus profondément, il faut remarquer que la rusticité des hypothèses fondatrices du régime Poole, ne permet pas une analyse correcte des phénomènes d'ionisation, propres à une paire. Nous savons, en particulier [7], qu'un calcul convenable des taux d'occupation des puits de potentiel, doit comprendre non seulement la détermination des probabilités d'excitation thermique, à laquelle se limitent les approches les plus courantes, mais aussi celle des sections de capture. Le déroulement du processus d'ionisation par le champ devrait, au minimum, pouvoir s'appuyer sur les considérations suivantes. (1) Sous champ nul, les probabilités d'occupation sont égales. (2) Lorsque le champ croît, le site 1 se vide plus rapidement que le second, car sa barrière de potentiel est réduite à la fois par le champ et par l'influence du site 2 (prépondérance du régime Poole). Il devrait donc exister un champ à partir duquel son taux d'occupation devient négligeable. (3) Au-delà, un effet $\mathrm{PF}$ modifié, selon l'équation (14b), devrait apparaître. En filigrane de cette discussion se profile le problème, à notre connaissance jamais abordé explicitement dans la littérature, de l'interaction entre puits vides et pleins. Contrairement aux apparences, il nécessitera une vaste discussion qu'il est prématuré d'entreprendre ici.

Nonobstant l'importance de ce dernier problème, ainsi que celui présenté par l'approche de Dussel et Böer, il est possible d'imaginer une manière plus simple de traduire les propositions (1) à (3) cidessus. Admettons pour plus de généralité, que le matériau contienne des accepteurs, de densité $N_{\mathrm{a}}$, situés à une profondeur telle qu'ils demeurent totalement occupés, quel que soit $F$. Alors l'équation $\mathrm{du}$ bilan détaillé s'écrit, en valeurs absolues : $n+N_{\mathrm{a}}=n_{1}+n_{2}, n_{1}$ et $n_{2}$ étant les densités d'élec- trons libérés des sites 1 et 2 . Supposons que les populations des sites 1 et 2 puissent être considérées comme indépendantes. Tel serait le cas si le calcul des probabilites d'occupation de ces sites ne faisait intervenir que les hauteurs des barrières de potentiel, que doivent surmonter les électrons pour s'échapper de leurs puits respectifs. Dans ces conditions, l'utilisation de la statistique de Fermi-Dirac conduit directement aux expressions $\left({ }^{1}\right)$ :

$$
\begin{aligned}
& n_{1}=\frac{N_{\mathrm{d}} / 2}{1+\exp \left(\frac{\Phi_{\mathrm{a}}^{\prime}-\Phi_{\mathrm{F}}-\Delta \Phi^{\prime}}{k T}\right)} \\
& n_{2}=\frac{N_{\mathrm{d}} / 2}{1+\exp \left(\frac{\Phi-\Phi_{\mathrm{F}}-\Delta \Phi^{\prime \prime}}{k T}\right)}
\end{aligned}
$$

$N_{\mathrm{d}}$ étant la densité totale des donneurs, $\Phi$ la profondeur effective des puits, $\Phi_{\mathrm{a}}^{\prime}=\Phi-\Phi^{\prime}$ la profondeur apparente du premier, $\Delta \Phi^{\prime}$ et $\Delta \Phi^{\prime \prime}$ les abaissements respectifs de barrière par le champ. Si on admet que la densité d'électrons dans la bande de conduction s'exprime par la fonction de Boltzmann $\left(n=N_{\mathrm{c}} \exp \left(-\Phi_{\mathrm{F}} / k T\right)\right)$, on obtient finalement, en valeurs relatives :

$$
\begin{gathered}
n_{\mathrm{r}}+\frac{s}{q}=\frac{s}{2}\left(\frac{1}{1+n_{\mathrm{r}} \exp \left(\frac{\Phi_{\mathrm{a}}^{\prime}-\Delta \Phi^{\prime}}{k T}\right)}+\right. \\
\left.+\frac{1}{1+n_{\mathrm{r}} \exp \left(\frac{\Phi-\Delta \Phi^{\prime \prime}}{k T}\right)}\right) .
\end{gathered}
$$

Dans cette équation $s$ est la densité relative des donneurs $s=N_{\mathrm{d}} / N_{\mathrm{c}}$, et $q$ est le taux de compensation $q=N_{\mathrm{d}} / N_{\mathrm{a}}$.

Nous n'étudierons pas plus avant l'équation (16) dans le cadre de cet article, souhaitant simplement donner ici quelques pistes de réflexion intéressantes.

\section{Conclusion.}

Les développements ci-dessus ont permis de clarifier un certain nombre de points. Accessoirement, la comparaison des approches de Hill et de Vollmann a mis en lumière les différences de conception que ces auteurs ont du régime PF, qu'ils associent au domaine des champs élevés. Notre présente contribution permet de prolonger l'analyse du modèle de base, adopté par tous les auteurs traitant du régime Poole. En particulier, notre approche mathématique du régime de type PF modifié, dû à l'ionisation du

(1) La rigueur exige que soit introduit $N_{\mathrm{d}} / 2$, bien que, comme indiqué au paragraphe 2 , les auteurs attachent peu d'importance au facteur $1 / 2$. 
second puits, nous a permis de préciser les limites de validité : d'une part du modèle hydrogénoïde de Hill, d'autre part du régime $P F$ usuel.

De même, notre traitement statistique, conduisant à l'équation (16), est beaucoup trop simple, dans la mesure où il exprime seulement la sommation des probabilités d'excitation thermique, par-dessus deux barrières de potentiel supposées indépendantes. Or on peut remarquer que, si le champ appliqué est faible, le franchissement de la barrière interne par l'électron du site 1 , ne signifie pas nécessairement qu'il soit libéré. En effet, lorsque le maximum de la barrière du site 1 est inférieur à celui du site 2 , le déplacement de cet électron à énergie constante l'amène au-dessous de ce dernier, plutôt que dans la bande de conduction. Il faudrait donc, en fait, conduire le calcul en déterminant séparément, à la manière de Dussel et Boër, les probabilités de capture et d'ionisation. Toutefois, contrairement à ces auteurs, le traitement devrait porter globalement sur la paire de sites, plutôt que sur le seul site 1 . La complexité du problème est alors extrême.

Néanmoins de tels calculs ne peuvent représenter qu'une approche trop grossièrement simplifiée du problème général des interactions, dans une population de donneurs. Nous avons, en effet, déjà longuement souligné l'étrangeté et l'insuffisance des hypothèses fondamentales du régime Poole. Nous savons qu'une résolution plus satisfaisante nécessiterait l'abandon du modèle de la paire isolée.

\section{Bibliographie}

[1] Poole, H. H., Phil. Mag. 32 (1916) 112-29.

[2] Poole, H. H., Phil. Mag. 34 (1917) 195.

[3] Poole, H. H., Phil. Mag. 42 (1921) 488.

[4] VollmanN, W., Phys. Status Solidi A 22 (1974) 195203.

[5] Pillonnet, A., Asch, G., Dufour, J., Lasjaunias, J. C., C. R. Acad. Sc. Paris 276B (1973) 639-41.

[6] Hill, R. M., Phil. Mag. 23 (1971) 59-86.
[7] Dussel, G. A., Böer, K. W., Phys. Status Solidi 39 (1970) 375-89.

[8] Feltz, A., Kahnt, H., Schirrmeister, F., J. Phys. Colloq. France C4 (1981) 935-8.

[9] Fritzsch, L., Bobe, W., Phys. Status Solidi B 58 (1973) K49-52.

[10] Simmons, J. G., Phys. Rev. 155 (1967) 657-60. 